\title{
Message from the Steering Committee Chair
}

Welcome to Zhangjiajie, China for the Tenth International Conference on Quality Software (QSIC 2010).

Software plays a vital role in our daily life. Unfortunately, it also fails to deliver according to promises. Many popular systems are renowned for their user-friendliness but notorious for their numerous faults. The QSIC conference provides a forum to bring together researchers and practitioners who work on improving the quality of software, enabling them to exchange ideas and present new results in this challenging area. It was first held in Hong Kong as an Asia-Pacific Conference. It then established itself as an international conference and was held in Dallas, Texas, USA; Braunschweig, Germany; Melbourne, Australia; Beijing, China; Portland, Oregon, USA; Oxford, UK; and Jeju, Korea. We are very pleased to be back in Asia this year to celebrate its tenth anniversary.

Over the last ten years, we have witnessed many new methodologies and techniques for solving the software crisis. On the other hand, we have also facing many new challenges in new applications, such as pervasive computing, wireless sensor networks, service-oriented architecture, cloud computing, and green IT. The quest for quality software still persists.

QSIC 2010 would not have been successful without the hard work of the organizers. We would like to thank the general chair Xicheng Lu for his leadership, and the program chairs Ji Wang, W.K. Chan, and Fei-Ching Kuo as well as their program committee for their hard work in creating an outstanding program that we will enjoy in the next two days. We are also grateful to the organizing chair Xiaoguang Mao, the IEEE Reliability Society liaison W. Eric Wong, the publicity and publication chair Xiaoying Bai, and the finance and registration chairs Yanjun Wen and Bo Jiang.

QSIC is also well known for our keynote speakers in the last ten years. They include internationally renowned researchers such as Lionel Briand, Joseph Goguen, David Harel, Rob Hierons, Ross Jeffery, Jeff Kramer, Edward A. Lee, David Parnas, Ray Paul, Mary Lou Soffa, C.V. Ramamoorthy, Gregg Rothermel, Anne Solvberg, Bhavani Thuraisingham, and Stephen Yau. We are honored to have to Paola Inverardi and Hans van Vliet for kindly agreeing to be our keynote speakers this year.

We have three international workshops being held in parallel with the main conference. We should extend our appreciation to all the workshop chairs, including Xiaoying Bai, Chih-Hung Chang, William C. Chu, Pao-Ann Hsiung, Qianxiang Wang, Chunyang Ye, and Zhenyu Zhang, as well as their respective teams.

QSIC 2010 is technically sponsored by IEEE Reliability Society and co-sponsored by National Laboratory for Parallel and Distributed Processing, China, and The University of Hong Kong, to whom we should extend our gratitude.

We should also thank all of you, who, as authors and participants, are helping us to assure the quality of this excellent conference. We hope you will find the conference enjoyable and fruitful.

Finally, we are pleased to announce that QSIC 2011 will be held in Madrid, Spain. We look forward to seeing all of you in Europe next year.

\section{T. H. Tse}

The University of Hong Kong

Pokfulam

Hong Kong

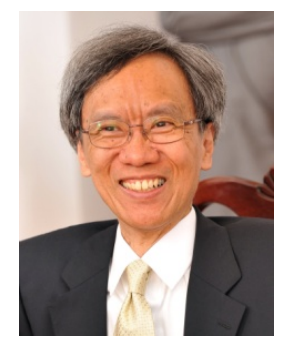

